\title{
Effect of submucosal injection in endoscopic papillectomy of ampullary tumor: Propensity-score matching analysis
}

United European Gastroenterology Journal 2018, Vol. 6(4) 576-585 (C) Author(s) 2017 Reprints and permissions: sagepub.co.uk/journalsPermissions.nav DOI: $10.1177 / 2050640617745459$ journals.sagepub.com/home/ueg @SAGE

\author{
Kwang Hyun Chung ${ }^{1}$, Sang Hyub Lee ${ }^{1}$, Jin Ho Choi ${ }^{1}$, Jinwoo Kang ${ }^{1}$, \\ Woo Hyun Paik ${ }^{1}$, Dong-Won Ahn ${ }^{2}$, ji Kon Ryu ${ }^{1}$ and Yong-Tae Kim ${ }^{1}$
}

\begin{abstract}
Background: The role of submucosal injection (SI) in endoscopic papillectomy (EP) is controversial. Objective: This study investigated the effects of SI before EP of ampullary tumors.

Methods: All patients who underwent initial curative EP at our institution between March 2006 and March 2014 were retrospectively recruited. The presence of residual tumor after three months, recurrence-free survival and post-procedural adverse events were compared between the SI group and non-injection (NI) group. Propensity-score matching was performed between the two groups to reduce potential selection bias and confounding.

Results: A total of 122 patients were included (SI: 26, NI: 96). Following propensity-score matching, 25 paired patients were selected. Residual tumor was not shown in the NI group, whereas seven (28.0\%) patients in the SI group had residual tumor $(p=0.010)$. The recurrence-free survival of the NI group was significantly longer than that of the SI group ( $p=0.036)$. Upon multivariate analysis, pathologic grade $(p=0.026)$ and SI $(p=0.033)$ were significantly related to recurrence-free survival. Post-procedural adverse events were not significantly different between the two groups.

Conclusion: SI before EP of ampullary tumor was related to more frequent residual tumor and shorter recurrence-free survival and did not reduce post-procedural adverse events.
\end{abstract}

\section{Keywords}

Endoscopic papillectomy, ampulla of Vater, neoplasm, injections, submucosal, neoplasm recurrence, local

Received: 1 August 2017; accepted: 6 November 2017

\section{Key summary}

- Endoscopic papillectomy is a well-established procedure for ampullary tumors of limited stage.

- The effect of submucosal injection before endoscopic papillectomy is controversial.

- This study showed more frequent residual tumor and shorter recurrence-free survival in the submucosal injection group.

- This study showed that submucosal injection did not reduce post-procedural adverse events.

\section{Introduction}

Ampullary adenomas are precancerous lesions that can transition into adenocarcinoma following an adenomacarcinoma sequence as in colorectal adenomas., Complete resection is required for ampullary adenomas and adenocarcinomas. Traditionally, surgical resection has been the standard treatment; however, endoscopic papillectomy is currently well accepted for treating ampullary adenoma alternating surgery with comparable efficacy and less morbidity. ${ }^{3-7}$ Even adenomas with
${ }^{1}$ Department of Internal Medicine and Liver Research Institute, Seoul National University College of Medicine, Seoul, Republic of Korea ${ }^{2}$ Department of Internal Medicine, Seoul Metropolitan Government Seoul National University Boramae Medical Center, Seoul, Republic of Korea

Corresponding author:

Sang Hyub Lee, Department of Internal Medicine and Liver Research Institute, Seoul National University Hospital, Seoul National University College of Medicine, 101, Daehak-ro, Jongno-gu, 03080, Seoul, Republic of Korea.

Email: gidoctor@snuh.org 
high-grade dysplasia or early-stage adenocarcinoma within an ampulla can be treated by endoscopic papillectomy in selected cases. ${ }^{6,8-10}$

Most gastrointestinal mucosal tumors, such as colonic polyps or early gastric cancers, can be lifted from the submucosal layer by injecting saline solution or diluted epinephrine solution into the submucosal layer beneath the lesion of the gastrointestinal wall. This submucosal injection (SI) is thought to yield wider resection margins and prevent perforation and hemorrhage and is therefore commonly conducted when performing endoscopic mucosal resection or endoscopic submucosal dissection of such tumors, ${ }^{11,12}$ as well as in endoscopic papillectomy. However, ampullary tumors do not just lie on the flat, plain gastrointestinal wall, but in the area in which three histologically and physiologically distinct anatomic structures (common bile duct, pancreatic duct, and duodenum) are combined. ${ }^{13}$ Moreover, the duodenal papilla itself lacks muscularis mucosae and a submucosal layer like the gallbladder and bile duct. ${ }^{14}$ Therefore, there is controversy regarding whether an SI should be performed before resection, ${ }^{15,16}$ and several endoscopists do not recommend it., However, this opinion is also based on theoretical evidence and personal experience, and lacks the data to support it. Nevertheless, few studies have investigated the effects of SI in endoscopic papillectomy. Therefore, this study compared the clinical outcomes of endoscopic papillectomy with and without SI in patients with ampullary adenomas or early-stage adenocarcinomas.

\section{Materials and methods}

\section{Patients}

Patients diagnosed with adenoma or adenocarcinoma of the ampulla of Vater at Seoul National University Hospital and who received endoscopic papillectomy between March 2006 and March 2014 were reviewed retrospectively. Patients found to have another type of tumor on the final pathologic result, who had received surgical or endoscopic papillectomy previously, or who had received endoscopic papillectomy as a palliative treatment were excluded. Patients diagnosed with familial adenomatous polyposis, who may have a higher recurrence rate compared with the general population, were also excluded, may confound the result. Using medical records, patients' demographic and clinical data including sex, age, presenting symptoms at diagnosis, estimated tumor size on endoscopic image and pretreatment pathologic grade based on endoscopic biopsy were reviewed. Details regarding the endoscopic papillectomy, pathologic findings describing the resected specimen and adverse events and follow-up results were reviewed. The study populations were followed until December 31, 2016. The study protocol was based on the Declaration of Helsinki and approved by the institutional review board of Seoul National University Hospital (IRB Approval Number/Date: H-1702-091-831/Feb-202017). The requirement for informed consent was waived.

\section{Endoscopic papillectomy}

All endoscopic procedures were performed by three experienced endoscopists (Y-TK, JKR and SHL). The entire endoscopic procedure was performed under fluoroscopic guidance using a standard side-view duodenoscope (JF-240, JF-260, TJF-240 and TJF-260; Olympus, Tokyo, Japan). Endoscopic retrograde cholangiopancreatogram was performed before endoscopic papillectomy to evaluate intraductal extension and determine resectability. Biliary or pancreatic stenting was performed when there was concern about inadequate biliary or pancreatic duct drainage after papillectomy. Sphincterotomy was conducted when quantification of the intraductal extension was needed or ampullary stenosis was a concern. Tumor resection was performed using a standard polypectomy snare with a blended electrosurgical current. SI was performed using a hypertonic saline mixed with epinephrine $(1: 50,000)$ and indigo carmine, mainly for lesions that could not be grasped with a snare because of lateral extension or to reduce the bleeding or perforation risk according to the performing endoscopist's judgment. Lesions that could not be removed by en bloc resection were resected in a piecemeal fashion. Argon plasma coagulation (APC) was used if residual tumor tissue was suspected on endoscopic image immediate after the snare excision. The resected specimens were captured with a basket and measured with a ruler.

The resection site was evaluated after each procedure to identify residual tumor or bleeding. If active bleeding was noted on the endoscopic image, hemostasis was performed as follows: epinephrine was sprayed on the bleeding site or injected adjacent to the submucosal space, cauterized with an electrical snare tip or needle knife, compressed with a retrieval balloon catheter, or hemoclipped.

\section{Definition of events and study outcome measurements}

The primary outcomes were residual tumor at three months after resection and recurrence-free survival. Following endoscopic papillectomy, all patients with a final pathologic result of adenocarcinoma or positive resection margin were recommended to receive subsequent surgical resection after being informed of the 
risks and benefits of surgery. For patients who did not receive subsequent surgical resection, follow-up endoscopy was performed three months after the procedure and then annually thereafter.

The secondary outcomes were procedure-related adverse events, such as pancreatitis, cholangitis, hemorrhage, perforation and ampullary stenosis. Pancreatitis was diagnosed when two of the following three features were noted: persistent abdominal pain characteristic of acute pancreatitis $>24 \mathrm{~h}$ after endoscopic papillectomy, serum amylase and/or lipase levels increased more than three times the upper limit of normal, and characteristic findings of acute pancreatitis on computed tomography (CT) scan. $^{17}$ Cholangitis was defined as the presence of abdominal pain, leukocytosis, and fever without any other infectious focus outside the hepatobiliary system that required antibiotic treatment within $24 \mathrm{~h} .{ }^{18}$ Significant bleeding was indicated by a decrease in the serum hemoglobin concentration $>2 \mathrm{mg} / \mathrm{dl}$ and a blood transfusion or hemostasis procedure required after the endoscopic papillectomy. ${ }^{19}$ Bowel perforation was diagnosed as the presence of free air in the abdominal cavity on post-procedural radiography or CT scan. Ampullary stenosis was diagnosed based on the radiological evidence of diffuse bile duct dilatation, as well as serum total bilirubin level elevated to more than twice the upper normal value.

\section{Statistical analyses}

Patients were divided into a non-injection (NI) group and an SI group. Data are shown as the number (\%) for categorical variables and the mean $( \pm \mathrm{SD})$ for continuous variables. To compare the characteristics of the study groups, the chi-squared test or Fisher's exact test was used for categorical variables, and the Student's $t$ test for the continuous variables, where appropriate. To compare the recurrence-free survival of patients, the Kaplan-Meier method and log-rank test were used. To identify the independent factors associated with these outcomes, a Cox proportional hazard model with a forward stepwise procedure was used for multivariate analysis. Two-sided $p$ values $<0.05$ were considered to be statistically significant. To better control for confounding in the selection of whether to perform SI and to reduce the number disparity between the two groups, we calculated the propensity-score by a logistic regression model, which represented the probability of each patient receiving SI. The two groups of patients were matched one to one by nearest-neighbor matching with a 0.3 -caliper distance. Variables that would affect the outcomes of endoscopic papillectomy were entered into the propensity-score matching, including age, sex, pretreatment pathology, and tumor size. The covariate imbalance in the unmatched and matched samples was measured using a standardized difference. Propensity scoring and matching were conducted using the MatchIt package (version 2.4-21) for $\mathrm{R}$ software ( $\mathrm{R}$ for Windows 3.3.4; The $\mathrm{R}$ Foundation for Statistical Computing, Vienna, Austria). ${ }^{20}$ Statistical analysis was performed with IBM SPSS Statistics version 22.0.0 for Windows (SPSS Inc, Chicago, IL, USA).

\section{Results}

\section{Patient characteristics}

During the study period, a total of 142 patients received endoscopic papillectomy. Among these, 20 were excluded from the analysis (Figure 1). Overall, 122 patients were included in the analysis (96 NI and 26 SI). The baseline characteristics of all eligible patients are presented in Table 1. Although not statistically significant, members of the NI group were slightly younger, included more males and had more tumors size $>2 \mathrm{~cm}$ than the SI group. Most patients had no symptoms, and the presenting symptoms did not differ between groups. Pretreatment pathology was also not significantly different.

Clinicopathological variables entered into the propensity-score matching analysis were age, sex, pretreatment pathology and tumor size. Overall, 25 paired patients were matched in both groups. The standardized mean differences in the clinicopathological characteristics between groups decreased (Table 1). Endoscopic ultrasonography was performed in 64 of $122(52.5 \%)$ patients in the original cohort (NI: 53/96 $(55.2 \%)$, SI: $11 / 26(42.3 \%), p=0.243)$ and 19 of 50 $(38 \%)$ patients in the propensity-score-matched cohort (NI: 9/25 (36\%), SI: 10/25 (40\%), $p=0.771)$.

\section{Endoscopic procedure details and pathological features of patients}

Endoscopic procedure details and pathological features following endoscopic papillectomy are described in Table 2. In the original cohort, sphincterotomy, piecemeal resection and endoscopic hemostasis were performed more frequently in the NI group, while endoscopic retrograde pancreatic drainage (ERPD) and cauterization were more common in the SI group; however, the difference was not significant. Endoscopic retrograde biliary drainage (ERBD) was performed more in the SI group than the NI group $(11(11.5 \%)$ vs. $7(26.9 \%), p=0.049)$. More patients in the NI group acquired a negative resection margin vertically and horizontally, although this difference was not significant. One tumor without pretreatment pathological exam, five tumors with low-grade adenoma, and 


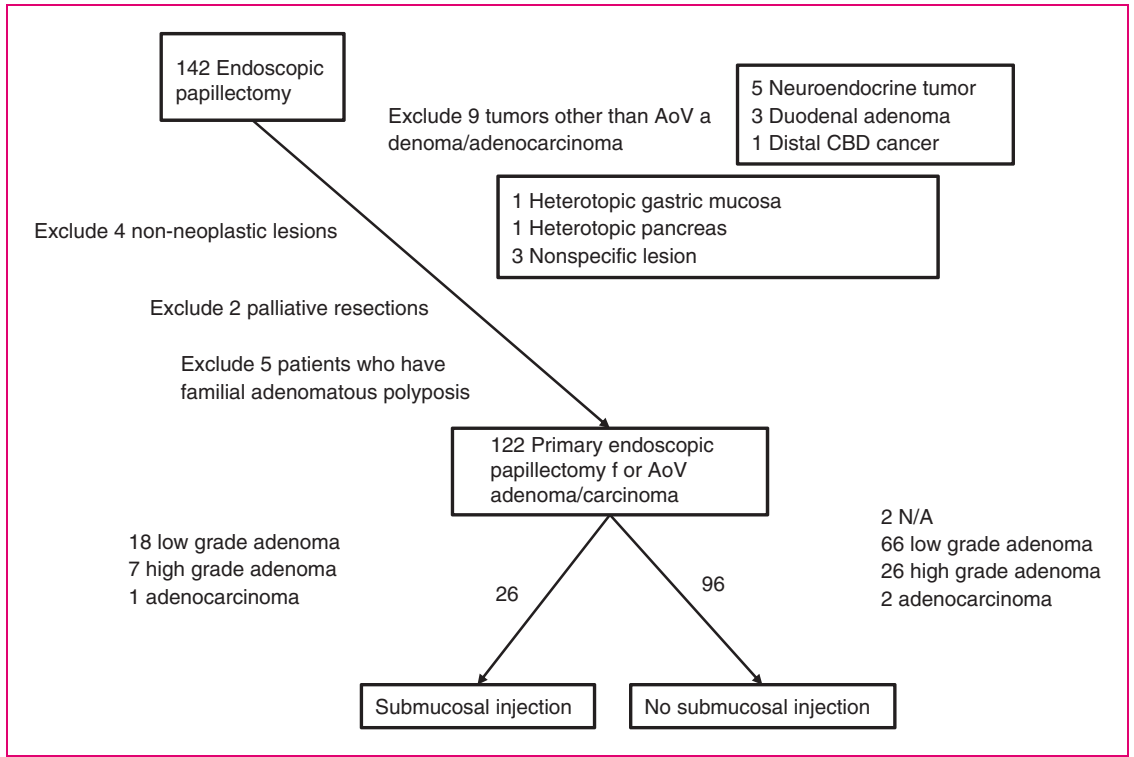

Figure 1. Flowchart of patient enrollment.

AoV: ampulla of Vater; CBD: common bile duct.

Table 1. Baseline characteristics of patients.

\begin{tabular}{|c|c|c|c|c|c|c|c|c|}
\hline \multirow[b]{2}{*}{ Variable } & \multicolumn{4}{|l|}{ Original cohort } & \multicolumn{4}{|c|}{ Propensity-score-matched cohort } \\
\hline & NI group & SI group & $p$ value & SMD & NI group & SI group & $p$ value & SMD \\
\hline$n$ & 96 & 26 & & & 25 & 25 & & \\
\hline Age (mean $\pm S D$ ) & $57.6 \pm 10.4$ & $60.3 \pm 10.3$ & 0.246 & 0.258 & $59.5 \pm 7.5$ & $59.3 \pm 9.2$ & 0.933 & 0.024 \\
\hline Sex (male) & $54(56.2 \%)$ & $11(42.3 \%)$ & 0.206 & 0.282 & $11(44.0 \%)$ & $10(40.0 \%)$ & 0.774 & 0.081 \\
\hline \multicolumn{3}{|c|}{ Pathology (prior to papillectomy) } & 0.849 & 0.230 & & & 0.507 & 0.334 \\
\hline $\mathrm{N} / \mathrm{A}$ & $2(2.1 \%)$ & $0(0.0 \%)$ & & & $0(0.0 \%)$ & $0(0.0 \%)$ & & \\
\hline LG adenoma & $66(68.8 \%)$ & $18(69.2 \%)$ & & & $19(76.0 \%)$ & $18(72.0 \%)$ & & \\
\hline HG adenoma & $26(27.1 \%)$ & $7(26.9 \%)$ & & & $5(20 \%)$ & $7(28.0 \%)$ & & \\
\hline Adenocarcinoma & $2(2.1 \%)$ & $1(3.8 \%)$ & & & $1(4.0 \%)$ & $0(0.0 \%)$ & & \\
\hline Tumor size & & & 0.082 & 0.565 & & & 0.948 & 0.093 \\
\hline$\sim 0.9 \mathrm{~cm}$ & $21(21.9 \%)$ & $6(23.1 \%)$ & & & $7(28.0 \%)$ & $6(24.0 \%)$ & & \\
\hline $1.0 \sim 1.9 \mathrm{~cm}$ & $48(50.0 \%)$ & $18(69.2 \%)$ & & & $16(64.0 \%)$ & $17(68.0 \%)$ & & \\
\hline $2.0 \mathrm{~cm} \sim$ & $27(28.1 \%)$ & $2(7.7 \%)$ & & & $2(8.0 \%)$ & $2(8.0 \%)$ & & \\
\hline \multicolumn{9}{|c|}{ Presenting symptom or sign } \\
\hline Jaundice & $1(1.0 \%)$ & $1(3.8 \%)$ & 0.382 & & $0(0.0 \%)$ & $1(4.0 \%)$ & 1.000 & \\
\hline P-duct dilatation & $4(4.2 \%)$ & $1(3.8 \%)$ & 1.000 & & $0(0.0 \%)$ & $1(4.0 \%)$ & 1.000 & \\
\hline B-duct dilatation & $1(1.0 \%)$ & $1(3.8 \%)$ & 0.382 & & $0(0.0 \%)$ & $1(4.0 \%)$ & 1.000 & \\
\hline Liver abscess & $1(1.0 \%)$ & $0(0.0 \%)$ & 1.000 & & $0(0.0 \%)$ & $0(0.0 \%)$ & & \\
\hline
\end{tabular}

B: bile; HG: high-grade; LG: low-grade; NI: non-injection; P: pancreatic; SD: standard deviation; SI: submucosal injection; SMD: standardized mean difference.

11 tumors with high-grade adenoma were identified as adenocarcinoma in the final pathological exam of the resected specimen; the proportion of each pathologic grade in both groups was not different, even based on the final pathological report.
There were also no significant differences in endoscopic procedure details and pathological features in the propensity-score-matched cohort, including the frequency of ERBD. A clear resection margin was more frequently achieved in the NI group $(52.0 \%$ vs. $40.0 \%)$; 
Table 2. Endoscopic procedure details and pathological features after endoscopic papillectomy.

\begin{tabular}{|c|c|c|c|c|c|c|}
\hline & \multicolumn{3}{|c|}{ Original cohort } & \multicolumn{3}{|c|}{ Propensity-score-matched cohort } \\
\hline & NI group & SI group & $p$ value & $\mathrm{NI}$ group & SI group & $p$ value \\
\hline$n$ & 96 & 26 & & 25 & 25 & \\
\hline \multicolumn{7}{|l|}{ Endoscopic procedure } \\
\hline $\begin{array}{l}\text { Sphincterotomy } \\
\text { (Preoperative/Postoperative) }\end{array}$ & $\begin{array}{c}28(29.2 \%) \\
(16 / 12)\end{array}$ & $\begin{array}{l}6(23.1 \%) \\
(5 / 1)\end{array}$ & 0.539 & $\begin{array}{l}6(24.0 \%) \\
(3 / 3)\end{array}$ & $\begin{array}{l}5(20.0 \%) \\
(4 / 1)\end{array}$ & 0.733 \\
\hline ERPD & $48(50.0 \%)$ & $17(65.4 \%)$ & 0.189 & $13(52.0 \%)$ & $16(64.0 \%)$ & 0.390 \\
\hline ERBD & $11(11.5 \%)$ & $7(26.9 \%)$ & 0.049 & $3(12.0 \%)$ & $7(28.0 \%)$ & 0.157 \\
\hline Cauterization (APC) & $22(22.9 \%)$ & $6(23.1 \%)$ & 1.000 & $4(16.0 \%)$ & $5(20.0 \%)$ & 1.000 \\
\hline Piecemeal resection & $13(13.5 \%)$ & $2(7.7 \%)$ & 0.522 & $2(8.0 \%)$ & $2(8.0 \%)$ & 1.000 \\
\hline Endoscopic hemostasis & $18(18.8 \%)$ & $2(7.7 \%)$ & 0.177 & $7(28.0 \%)$ & $2(8.0 \%)$ & 0.138 \\
\hline Epi. spray/injection & $11(11.5 \%)$ & $0(0.0 \%)$ & 0.070 & $5(20.0 \%)$ & $0(0.0 \%)$ & 0.050 \\
\hline Electrocauterization & $6(6.2 \%)$ & $2(7.7 \%)$ & 0.678 & $2(8.0 \%)$ & $2(8.0 \%)$ & 1.000 \\
\hline Balloon compression & $2(2.1 \%)$ & $0(0.0 \%)$ & 1.000 & $1(4.0 \%)$ & $0(0.0 \%)$ & 1.000 \\
\hline Hemoclipping & $2(2.1 \%)$ & $0(0.0 \%)$ & 1.000 & $0(0.0 \%)$ & $0(0.0 \%)$ & - \\
\hline \multicolumn{7}{|l|}{ Pathologic finding } \\
\hline Deep resection margin & & & 0.664 & & & 0.904 \\
\hline Negative & $62(64.6 \%)$ & $16(61.5 \%)$ & & $15(60.0 \%)$ & $15(60.0 \%)$ & \\
\hline Positive & $12(12.5 \%)$ & $5(19.2 \%)$ & & $4(16.0 \%)$ & $5(20.0 \%)$ & \\
\hline Uncheckable & $22(22.9 \%)$ & $5(19.2 \%)$ & & $6(24.0 \%)$ & $5(20.0 \%)$ & \\
\hline Lateral resection margin & & & 0.202 & & & 0.265 \\
\hline Negative & $55(57.3 \%)$ & $13(50.0 \%)$ & & $15(60.0 \%)$ & $12(48.0 \%)$ & \\
\hline Positive & $18(18.8 \%)$ & $9(34.6 \%)$ & & $4(16.0 \%)$ & $9(36.0 \%)$ & \\
\hline Uncheckable & $23(24.0 \%)$ & $4(15.4 \%)$ & & $6(24.0 \%)$ & $4(16.0 \%)$ & \\
\hline Any resection margin & & & 0.260 & & & 0.323 \\
\hline Negative & $49(51.0 \%)$ & $11(42.3 \%)$ & & $13(52.0 \%)$ & $10(40.0 \%)$ & \\
\hline Positive & $25(26.0 \%)$ & $11(42.3 \%)$ & & $6(24.0 \%)$ & $11(44.0 \%)$ & \\
\hline Uncheckable & $22(22.9 \%)$ & $4(15.4 \%)$ & & $6(24.0 \%)$ & $4(16.0 \%)$ & \\
\hline Pathologic finding after resection & & & 0.747 & & & 0.931 \\
\hline Low grade adenoma & $56(58.3 \%)$ & $16(61.5 \%)$ & & $15(60.0 \%)$ & $16(64.0 \%)$ & \\
\hline High grade adenoma & $25(26.0 \%)$ & $5(19.2 \%)$ & & $5(20.0 \%)$ & $5(20.0 \%)$ & \\
\hline Adenocarcinoma & $15(15.6 \%)$ & $5(19.2 \%)$ & & $5(20.0 \%)$ & $4(16.0 \%)$ & \\
\hline
\end{tabular}

Epi.: epinephrine; ERBD: endoscopic retrograde biliary drainage; ERPD: endoscopic retrograde pancreatic drainage; NI: non-injection; SI: submucosal injection.

however, this difference was not significant. In the final pathologic report, the proportion of high-grade adenoma was the same and the NI group had one more adenocarcinoma, but this difference was not significant.

\section{Primary outcomes}

Residual tumor was more frequent in the SI group $(26.9 \%)$ than the NI group $(8.3 \%)(p=0.010)$ in the original cohort. Six $(6.2 \%)$ patients in the NI group and three $(11.5 \%)$ in the SI group were lost to followup at three months after endoscopic papillectomy (Table 3). There was no significant difference in the residual tumor rate depending on the use of APC
$(4 / 28(14.3 \%)$ with APC and 11/94 (11.7\%) without APC, $p=0.746)$. The recurrence-free survival was not significantly different between the groups (Figure 2(a), Table 4). Factors related to shorter recurrence-free survival were piecemeal resection $(p=0.033)$ and advanced histology both in the pretreatment $(p=0.017)$ and final $(p=0.001)$ (Table 4). Upon multivariate analysis, advanced histology was a significant factor only for shorter recurrence-free survival (Table 5).

In the propensity-score-matched cohort, residual tumor was not observed in the NI group and seven $(28.0 \%)$ patients in the SI group had residual tumor $(p=0.010)$. The proportion of patients who underwent subsequent surgery or were lost to follow-up was 
Table 3. Follow-up results and adverse events at three months after endoscopic papillectomy.

\begin{tabular}{|c|c|c|c|c|c|c|}
\hline & \multicolumn{3}{|c|}{ Original cohort } & \multicolumn{3}{|c|}{ Propensity-score-matched cohort } \\
\hline & $\mathrm{NI}$ group & SI group & $p$ value & $\mathrm{NI}$ group & SI group & $p$ value \\
\hline$n$ & 96 & 26 & & 25 & 25 & \\
\hline \multicolumn{7}{|c|}{ Follow-up result at three months after endoscopic papillectomy } \\
\hline Subsequent surgery & $11(11.5 \%)$ & $1(3.8 \%)$ & 0.458 & $3(12.0 \%)$ & $1(4.0 \%)$ & 0.609 \\
\hline Follow-up loss & $6(6.2 \%)$ & $3(11.5 \%)$ & 0.400 & $3(12.0 \%)$ & $3(12.0 \%)$ & 1.000 \\
\hline Residual tumor & $8(8.3 \%)$ & $7(26.9 \%)$ & 0.010 & $0(0.0 \%)$ & $7(28.0 \%)$ & 0.010 \\
\hline No evidence of residual tumor & $71(74.0 \%)$ & $15(57.7 \%)$ & 0.107 & $19(76.0 \%)$ & $14(56.0 \%)$ & 0.136 \\
\hline \multicolumn{7}{|l|}{ Procedure-related adverse event } \\
\hline Pancreatitis & $10(10.4 \%)$ & $7(26.9 \%)$ & 0.031 & $2(8.0 \%)$ & $7(28.0 \%)$ & 0.138 \\
\hline Cholangitis & $4(4.2 \%)$ & $1(3.8 \%)$ & 1.000 & $0(0.0 \%)$ & $1(4.0 \%)$ & 1.000 \\
\hline Bleeding & $5(5.2 \%)$ & $5(19.2 \%)$ & 0.021 & $1(4.0 \%)$ & $5(20.0 \%)$ & 0.189 \\
\hline Perforation & $0(0.0 \%)$ & $2(7.7 \%)$ & 0.044 & $0(0.0 \%)$ & $2(8.0 \%)$ & 0.490 \\
\hline Ampullary stenosis & $3(3.1 \%)$ & $3(11.5 \%)$ & 0.110 & $0(0.0 \%)$ & $3(12.0 \%)$ & 0.235 \\
\hline
\end{tabular}

NI: non-injection; SI: submucosal injection.

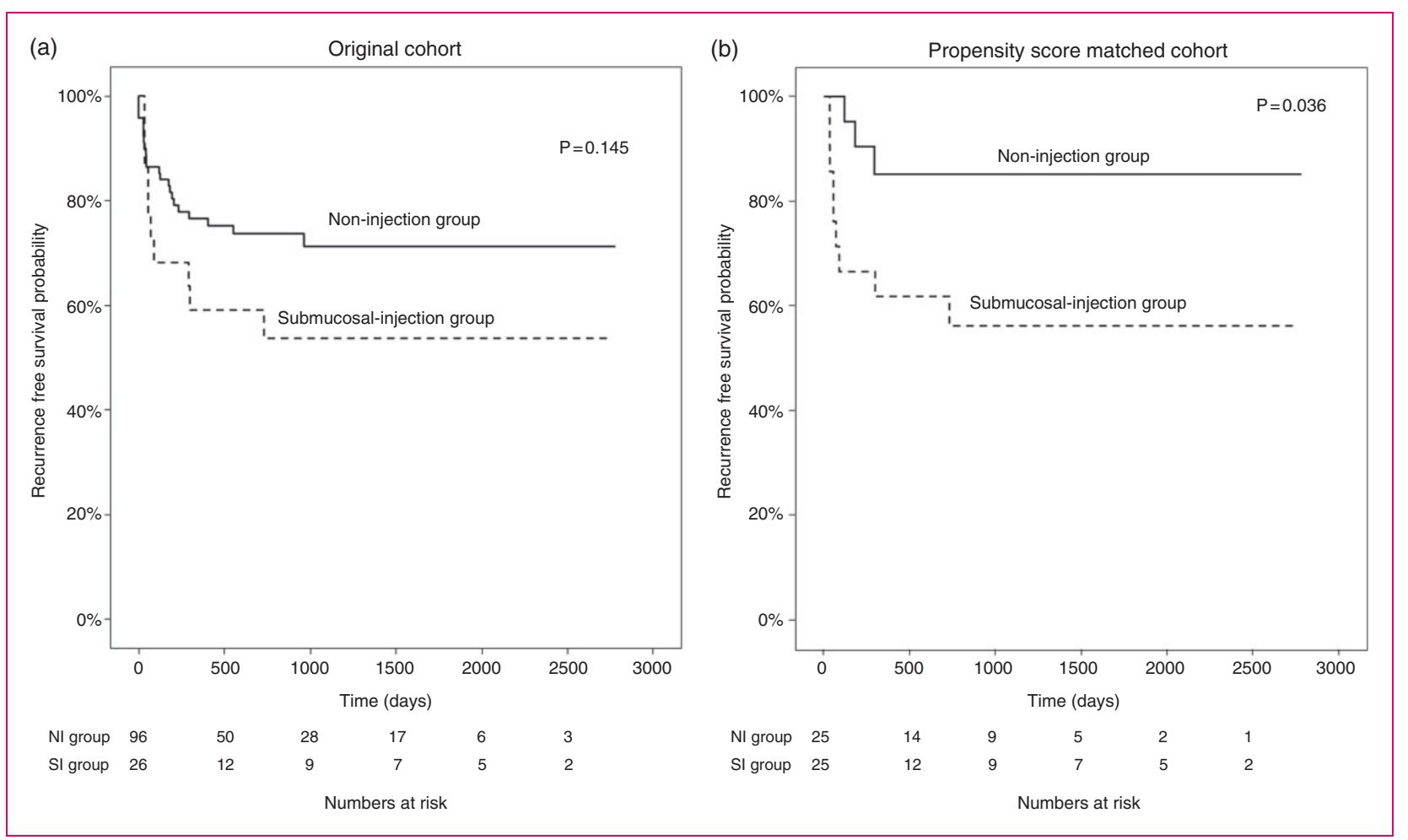

Figure 2. Kaplan-Meier estimation of recurrence-free survival for the non-injection (NI) group and the submucosal injection (SI) group. (a) The recurrence-free survival was not significantly different between the two groups $(p=0.145)$ in the original cohort. Non-injection group (solid line); submucosal injection group (broken line). (b) The recurrence-free survival rate of the non-injection group was significantly longer than that of the submucosal injection group $(p=0.036)$ in the propensity-score-matched cohort. The non-injection group (solid line); submucosal injection group (broken line).

similar in both groups (Table 3). Eleven (11.5\%) patients in the NI group (four with positive resection margins, seven confirmed to have adenocarcinoma) and one $(3.8 \%)$ in the SI group (confirmed to have adenocarcinoma) underwent subsequent pylorus-preserving pancreaticoduodenectomy. The NI group had a significantly longer recurrence-free survival rate than the SI group ( $p=0.036$ ) (Figure 2(b), Table 4). Upon univariate analysis, the only factor significantly related to shorter recurrence-free survival other than SI was 
Table 4. Univariate analysis of factors related to recurrence-free survival.

\begin{tabular}{|c|c|c|c|c|c|c|c|c|}
\hline & \multicolumn{4}{|c|}{ Original cohort } & \multicolumn{4}{|c|}{ Propensity-score-matched cohort } \\
\hline & $n$ & Mean (days) & $95 \% \mathrm{Cl}$ & $p$ value & $\mathrm{n}$ & Mean (days) & $95 \% \mathrm{Cl}$ & $p$ value \\
\hline \multicolumn{4}{|l|}{ Age } & 0.543 & & & & 0.303 \\
\hline$<65$ & 88 & 2015.1 & $1743.0-2287.3$ & & 36 & 2147.4 & $1737.0-2557.9$ & \\
\hline$\geq 65$ & 34 & 1611.4 & $1185.1-2037.7$ & & 14 & 1564.8 & $916.5-2213.2$ & \\
\hline \multicolumn{4}{|l|}{ Sex (M/F) } & 0.877 & & & & 0.458 \\
\hline Male & 65 & 1978.0 & $1666.8-2289.1$ & & 21 & 2182.2 & $1664.5-2699.9$ & \\
\hline Female & 57 & 1746.8 & $1408.1-2085.4$ & & 29 & 1668.6 & $1222.1-2115.0$ & \\
\hline \multicolumn{4}{|l|}{ Submucosal injection } & 0.145 & & & & 0.036 \\
\hline No & 96 & 2040.7 & $1780.4-2301.0$ & & 25 & 2398.3 & $1997.6-2798.9$ & \\
\hline Yes & 26 & 1566.6 & $1019.4-2113.9$ & & 25 & 1627.3 & $1067.1-2187.6$ & \\
\hline \multicolumn{4}{|c|}{ Argon plasma coagulation } & 0.815 & & & & 0.852 \\
\hline No & 94 & 1920.0 & $1644.5-2195.5$ & & 41 & 1989.2 & $1577.9-2400.6$ & \\
\hline Yes & 28 & 1850.0 & $1408.5-2291.6$ & & 9 & 1786.8 & $1090.3-2483.2$ & \\
\hline \multicolumn{4}{|l|}{ Piecemeal resection } & 0.033 & & & & 0.108 \\
\hline No & 107 & 2029.8 & $1783.3-2276.3$ & & 46 & 2092.0 & $1724.2-2459.8$ & \\
\hline Yes & 15 & 941.3 & $439.8-1442.8$ & & 4 & 721.7 & $0.0-1687.1$ & \\
\hline \multicolumn{4}{|c|}{ Pretreatment pathology } & 0.017 & & & & 0.006 \\
\hline LG adenoma & 84 & 2143.2 & $1885.5-2400.8$ & & 37 & 2219.5 & $1862.1-2576.9$ & \\
\hline HG adenoma & 33 & 1336.3 & $810.6-1862.0$ & & 12 & 1010.4 & $79.9-1940.8$ & \\
\hline Adenocarcinoma & 3 & 527.0 & $205.2-828.8$ & & 1 & $\mathrm{~N} / \mathrm{A}$ & & \\
\hline \multicolumn{4}{|l|}{ Final pathology } & 0.001 & & & & 0.022 \\
\hline LG adenoma & 72 & 2217.3 & $1945.7-2479.8$ & & 31 & 2291.9 & $1919.2-2664.6$ & \\
\hline HG adenoma & 30 & 1196.7 & $684.3-1709.1$ & & 10 & 1099.3 & $273.1-1925.5$ & \\
\hline Adenocarcinoma & 20 & 1945.6 & $992.7-1910.3$ & & 9 & 1462.3 & $675.1-2249.4$ & \\
\hline \multicolumn{4}{|l|}{ Tumor size } & 0.337 & & & & 0.958 \\
\hline$\sim 0.9 \mathrm{~cm}$ & 27 & 1319.0 & $903.3-1734.7$ & & 13 & 1699.0 & $1160.0-2238.0$ & \\
\hline $1.0 \sim 1.9 \mathrm{~cm}$ & 66 & 2032.1 & $1719.8-2344.4$ & & 33 & 1971.5 & $1504.7-2438.3$ & \\
\hline $2.0 \mathrm{~cm} \sim$ & 29 & 2117.7 & $1654.0-2581.3$ & & 4 & 948.8 & $515.5-1382.0$ & \\
\hline \multicolumn{4}{|l|}{ Deep resection margin } & 0.424 & & & & 0.879 \\
\hline Not involved & 78 & 1852.9 & $1548.6-2157.2$ & & 30 & 2003.3 & $1528.1-2478.6$ & \\
\hline Involved & 17 & 2169.7 & $1564.7-2774.7$ & & 9 & 2103.9 & $1300.6-2907.1$ & \\
\hline \multicolumn{4}{|c|}{ Lateral resection margin } & 0.050 & & & & 0.265 \\
\hline Not involved & 68 & 2116.3 & $1823.4-2409.2$ & & 27 & 2196.3 & $1744.5-2648.2$ & \\
\hline Involved & 27 & 1330.9 & $805.1-1856.6$ & & 13 & 1562.9 & $833.0-2292.8$ & \\
\hline \multicolumn{4}{|l|}{ Any resection margin } & 0.330 & & & & 0.297 \\
\hline Not involved & 60 & 2031.6 & $1715.9-2347.3$ & & 23 & 2219.0 & $1744.4-2693.6$ & \\
\hline Involved & 36 & 1696.1 & $1203.8-2188.4$ & & 17 & 1805.5 & $1129.0-2482.0$ & \\
\hline
\end{tabular}

M: male; F: female; Cl: confidence interval; HG: high-grade; LG: low-grade.

advanced tumor histology. Upon multivariate analysis, SI $(p=0.033)$ and advanced histology $(p=0.026)$ were still significant factors related to shorter recurrence-free survival (Table 5).

\section{Secondary outcomes}

After the procedure, pancreatitis, bleeding, and perforation were significantly more common in the SI group than the NI group. Pancreatitis occurred in fewer patients who underwent ERPD (11/57, 19.3\% vs. 6/ $65,9.2 \%)$; however, this difference was not statistically significant $(p=0.109)$. Although not statistically significant, ampullary stenosis was also more common in the SI group. Adverse events were more frequent in the SI group, even in the propensity-score-matched cohort; however, the difference was reduced and not statistically significant (Table 3). Residual or recurrent tumor 
Table 5. Multivariate analysis of factors related to recurrence-free survival.

\begin{tabular}{|c|c|c|c|c|c|c|}
\hline & \multicolumn{3}{|c|}{ Original cohort } & \multicolumn{3}{|c|}{ Propensity-score-matched cohort } \\
\hline & $p$ value & $\mathrm{HR}$ & $95 \% \mathrm{Cl}$ & $p$ value & $\mathrm{HR}$ & $95 \% \mathrm{Cl}$ \\
\hline Submucosal injection & 0.055 & 2.095 & $0.986-4.454$ & 0.033 & 4.217 & $1.125-15.803$ \\
\hline Piecemeal resection & 0.051 & 2.399 & $0.996-5.781$ & & & \\
\hline Final pathology & 0.002 & & & 0.026 & & \\
\hline Low-grade adenoma & & 1 & & & 1 & \\
\hline High-grade adenoma & 0.001 & 3.440 & $1.643-7.201$ & 0.007 & 5.349 & $1.566-18.267$ \\
\hline Adenocarcinoma & 0.939 & 0.951 & $0.265-3.412$ & 0.675 & 1.583 & $0.184-13.604$ \\
\hline
\end{tabular}

$\mathrm{Cl}$, confidence interval; HR: hazard ratio.

was identified in 30 patients during follow-up. Of these patients, 13 were re-treated with APC, six with snare papillectomy, six with multiple cold forceps biopsy, four with pylorus-preserving pancreaticoduodenectomy (all had recurred as a carcinoma), and one patient refused further treatment. A total of 11 of 30 patients eventually received pylorus-preserving pancreaticoduodenectomy during follow-up.

\section{Discussion}

There is a debate over whether to perform SI before endoscopic papillectomy of ampullary tumor and there is also a little evidence for not recommending it. ${ }^{3,16,21-23}$ This study showed that SI was related to more frequent residual tumor and shorter recurrence-free survival. Theoretically, SI lifts the tumor from the wall to secure a wider resection margin and prevent perforation and bleeding. Contrary to expectations, the SI group did not acquire a more negative margin than the NI group. Rather, the negative margin rate was slightly higher in the NI group than in the SI group, although this difference was not significant. Moreover, residual tumor was more frequent and recurrence-free survival was significantly shorter in the SI group than in the NI group. Furthermore, the procedure-related adverse events were more common in the SI group than in the NI group, although the differences were not significant.

It is well known that SI when performing endoscopic mucosal resection of colonic polyps can create a fluid cushion between the lesion and deep layers of the gastrointestinal wall. This allows safe and complete en bloc resection of the lesion and prevents adverse events such as perforation and bleeding. ${ }^{11,12,24}$ However, in ampullary tumors, it may not be applicable because of differences in histological features between the colonic mucosa and duodenal papilla. SI cannot lift the common bile duct and pancreatic duct, which pass through the duodenal wall. It could possibly lift the tumor confined to the duodenal surface, but tumors within the ampullary lumen (intra-ampullary), or involving the walls of the distal ends of the pancreatic duct and/or common bile duct could not be lifted, and would rather be hidden behind the swollen periampullary tissue. Moreover, SI could blur the margins of the tumor and make it difficult to grasp the entire tumor tissue with a snare. ${ }^{25}$ Therefore, SI could interfere with complete resection of the tumor, which may be expressed in our study as a more positive resection margin in the tumor specimen and more frequent residual tumors in the SI group.

There is no standard equipment or technique for endoscopic papillectomy, only an approximate method is described ${ }^{16,25}$ and little is known about the role of SI. In early case series of endoscopic papillectomies, SI was routinely performed, and whether being lifted or not was used as an indicator of invasiveness; therefore, the effects of SI were not evaluable. ${ }^{21}$ In another study, 70 endoscopic papillectomy cases were analyzed, suggesting that SI did not reduce the risk of delayed bleeding and perforation and the effect of SI on tumor recurrence was not evaluated. ${ }^{26}$ In another study, 106 cases of endoscopic papillectomy showed recurrence in only $15 \%$ of patients, although none of the patients underwent SI. ${ }^{3}$ In one small study, SI was not significantly associated with complete resection rate. ${ }^{27}$ Recently, a prospective multicenter study was conducted to investigate the effects of SI in endoscopic papillectomy. In this study, more negative resection margin was achieved in patients who did not receive SI $(21 / 26)$ than in those who did receive SI $(12 / 24) \quad(p=0.02)$. However, unlike our study, it included only benign tumors. The recurrence rate at one month and 12 months was not significantly different despite the initial difference in the rate of positive resection margin. ${ }^{28}$

SI was expected to reduce bleeding; however, our study results showed that SI is not an effective method to reduce bleeding. Previous studies have also reported that SI did not reduce bleeding, ${ }^{26}$ which is in good agreement with our results. In our study, perforation occurred in two patients and both patients were 
in the SI group. Bowel perforation is a relatively uncommon complication after endoscopic papillectomy; therefore, there were no data available for this. Contrary to our findings, retroperitoneal perforation was previously reported in patients who did not undergo SI. ${ }^{26,28,29}$ Perhaps SI could encourage wider resection, which leads to diminished protective effects of SI and may explain the higher rate of bleeding and perforation in the SI group. Pancreatitis was also more common in the SI group, although there was no significant difference after propensity-score matching. These results were similar to those of a previous study and suggest that the swollen periampullary duodenal mucosa and injected solution could block the pancreatic ductal orifice. ${ }^{28}$ Moreover, the injected solution may directly cause pancreatic tissue damage, which could induce pancreatitis.

In some special cases, SI could be helpful such as for large lateral spreading tumors spreading out of the papilla. In this case, tumor components spreading out of the papilla can be removed with SI and tumor components within the papilla can be removed without SI. ${ }^{30}$ Our study did not include such large tumors, and large tumors up to $3 \mathrm{~cm}$ were resected without SI or any special technique. In our study, only two tumors larger than $2 \mathrm{~cm}$ in diameter were included in the SI group, $2 \mathrm{~cm}$ and $2.3 \mathrm{~cm}$, respectively.

It should be noted that this study was limited in that it was conducted in a retrospective fashion and the selection of SI was not randomized.

Unfortunately, only a few patients in our study had undergone endoscopic ultrasonography, which could be useful to evaluate intraductal invasion of the tumor. $^{31,32}$ Moreover, evaluation of the resection margin was not performed in many patients, and determination of whether to conduct SI could be influenced by unrecognized factors or physician's preference. A relatively large number of patients were lost to follow-up at less than three months (approximately $10 \%$ ) after the procedure, which could have caused bias. ${ }^{33}$ However, the baseline characteristics of patients were not significantly different between the two groups including the frequency of endoscopic sonography, and we conducted propensity-score matching to reduce bias during patient selection. The difference in frequency of residual tumor was significant, even after the propensity-score matching, and the difference in recurrencefree survival became more prominent after matching. Considering the slow doubling time of adenoma and early ampullary adenocarcinoma, we expect that the follow-up loss of patients within three months of the procedure may not be from the difference in clinical outcomes or acute deterioration of patients. Moreover, the dropout rates of both groups were similar, which may not seriously affect the study outcome.
Our study is significant because it is the first study to show a worse outcome of endoscopic papillectomy with SI than without SI. We have included a relatively large number of patients at a single center and included patients with ampullary adenocarcinoma, as well as those with adenoma, which reflects actual clinical practice. Many different factors that could affect the clinical outcome were compared between groups, and no factors were significantly different between groups after propensity-score matching.

Whether to perform SI during endoscopic papillectomy is still controversial and needs to be elucidated. We found that SI was related to more frequent residual tumor and shorter recurrence-free survival. Protective effects of SI for post-procedural bleeding or perforation were not seen, and it instead seemed to be harmful, although the results were not statistically significant. In conclusion, SI before endoscopic papillectomy is not recommended because of its impact on residual tumor, tumor recurrence, and post-procedural adverse events.

\section{Acknowledgments}

We appreciate the statistical advice provided by the Medical Research Collaborating Center at the Seoul National University College of Medicine and the Seoul National University Hospital.

The abstract of this paper was accepted as an oral presentation and awarded a travel grant during the 25th United Gastroenterology (UEG) week held in Barcelona, Spain, and was presented 1 November 2017.

\section{Declaration of Conflicting Interests}

None declared.

\section{Ethics approval}

The study protocol was based on the Declaration of Helsinki and approved by the institutional review board of Seoul National University Hospital (IRB Approval Number/Date: H-1702-091-831/Feb-20-2017).

\section{Funding}

This research received no specific grant from any funding agency in the public, commercial, or not-for-profit sectors.

\section{Informed consent}

The requirement for informed consent for this study was waived.

\section{References}

1. Seifert E, Schulte F and Stolte M. Adenoma and carcinoma of the duodenum and papilla of Vater: A clinicopathologic study. Am J Gastroenterol 1992; 87: 37-42. 
2. Stolte $\mathrm{M}$ and Pscherer C. Adenoma-carcinoma sequence in the papilla of Vater. Scand J Gastroenterol 1996; 31: 376-382.

3. Bohnacker S, Seitz U, Nguyen D, et al. Endoscopic resection of benign tumors of the duodenal papilla without and with intraductal growth. Gastrointest Endosc 2005; 62: $551-560$

4. Irani S, Arai A, Ayub K, et al. Papillectomy for ampullary neoplasm: Results of a single referral center over a 10-year period. Gastrointest Endosc 2009; 70: 923-932.

5. Napoléon B, Gincul R, Ponchon T, et al. Endoscopic papillectomy for early ampullary tumors: Long-term results from a large multicenter prospective study. Endoscopy 2014; 46: 127-134.

6. Moon JH, Choi HJ and Lee YN. Current status of endoscopic papillectomy for ampullary tumors. Gut Liver 2014; 8: 598-604.

7. Ahn DW, Ryu JK, Kim J, et al. Endoscopic papillectomy for benign ampullary neoplasms: How can treatment outcome be predicted? Gut Liver 2013; 7: 239-245.

8. Yoon SM, Kim MH, Kim MJ, et al. Focal early stage cancer in ampullary adenoma: Surgery or endoscopic papillectomy? Gastrointest Endosc 2007; 66: 701-707.

9. Harano M, Ryozawa S, Iwano H, et al. Clinical impact of endoscopic papillectomy for benign-malignant borderline lesions of the major duodenal papilla. $J$ Hepatobiliary Pancreat Sci 2011; 18: 190-194.

10. Alvarez-Sanchez MV, Oria I, Luna OB, et al. Can endoscopic papillectomy be curative for early ampullary adenocarcinoma of the ampulla of Vater? Surg Endosc 2017; 31: 1564-1572.

11. Yandrapu H, Desai M, Siddique S, et al. Normal saline solution versus other viscous solutions for submucosal injection during endoscopic mucosal resection: A systematic review and meta-analysis. Gastrointest Endosc 2017; 85: 693-699.

12. Ferlitsch M, Moss A, Hassan C, et al. Colorectal polypectomy and endoscopic mucosal resection (EMR): European Society of Gastrointestinal Endoscopy (ESGE) Clinical Guideline. Endoscopy 2017; 49: 270-297.

13. Adsay V, Ohike N, Tajiri T, et al. Ampullary region carcinomas: Definition and site specific classification with delineation of four clinicopathologically and prognostically distinct subsets in an analysis of 249 cases. Am J Surg Pathol 2012; 36: 1592-1608.

14. Horiguchi S and Kamisawa T. Major duodenal papilla and its normal anatomy. Dig Surg 2010; 27: 90-93.

15. Menees SB, Schoenfeld P, Kim HM, et al. A survey of ampullectomy practices. World J Gastroenterol 2009; 15 : 3486-3492.

16. De Palma GD. Endoscopic papillectomy: Indications, techniques, and results. World J Gastroenterol 2014; 20 : 1537-1543.

17. Banks PA, Freeman ML and Practice Parameters Committee of the American College of Gastroenterology. Practice guidelines in acute pancreatitis. Am J Gastroenterol 2006; 101: 2379-2400.
18. Kapral C, Duller C, Wewalka F, et al. Case volume and outcome of endoscopic retrograde cholangiopancreatography: Results of a nationwide Austrian benchmarking project. Endoscopy 2008; 40: 625-630.

19. Coté GA and Sherman S. Advances in pancreatobiliary endoscopy. Curr Opin Gastroenterol 2010; 26: 429-435.

20. Ho D, Imai K, King G, et al. MatchIt: Nonparametric preprocessing for parametric causal inference. J Stat Softw 2011; 42: 1-28.

21. Desilets DJ, Dy RM, Ku PM, et al. Endoscopic management of tumors of the major duodenal papilla: Refined techniques to improve outcome and avoid complications. Gastrointest Endosc 2001; 54: 202-208.

22. Ardengh JC, Kemp R, Lima-Filho ÉR, et al. Endoscopic papillectomy: The limits of the indication, technique and results. World J Gastrointest Endosc 2015; 7: 987-994.

23. Bohnacker S, Soehendra N, Maguchi H, et al. Endoscopic resection of benign tumors of the papilla of Vater. Endoscopy 2006; 38: 521-525.

24. Lee SH, Lee KS, Park YS, et al. Submucosal salineepinephrine injection in colon polypectomy: Appropriate indication. Hepatogastroenterology 2008; 55: 1589-1593.

25. Aiura K, Imaeda H, Kitajima M, et al. Balloon-catheterassisted endoscopic snare papillectomy for benign tumors of the major duodenal papilla. Gastrointest Endosc 2003; 57: 743-747.

26. Cheng CL, Sherman S, Fogel EL, et al. Endoscopic snare papillectomy for tumors of the duodenal papillae. Gastrointest Endosc 2004; 60: 757-764.

27. Han J, Lee SK, Park DH, et al. Treatment outcome after endoscopic papillectomy of tumors of the major duodenal papilla [article in Korean]. Korean J Gastroenterol 2005; 46: 110-119.

28. Hyun JJ, Lee TH, Park JS, et al. A prospective multicenter study of submucosal injection to improve endoscopic snare papillectomy for ampullary adenoma. Gastrointest Endosc 2017; 85: 746-755.

29. Maguchi H, Takahashi K, Katanuma A, et al. Indication of endoscopic papillectomy for tumors of the papilla of Vater and its problems. Dig Endosc 2003; 15: S33-S35.

30. Klein A, Tutticci N and Bourke MJ. Endoscopic resection of advanced and laterally spreading duodenal papillary tumors. Dig Endosc 2016; 28: 121-130.

31. Trikudanathan G, Njei B, Attam R, et al. Staging accuracy of ampullary tumors by endoscopic ultrasound: Meta-analysis and systematic review. Dig Endosc 2014; 26: 617-626.

32. Moon JH. Endoscopic diagnosis of ampullary tumors using conventional endoscopic ultrasonography and intraductal ultrasonography in the era of endoscopic papillectomy: Advantages and limitations. Clin Endosc 2014; 47: 127-128.

33. Dettori JR. Loss to follow-up. Evid Based Spine Care J 2011; 2: 7-10. 